\title{
Validation of 2 noninvasive, markerless reconstruction techniques in biplane high-speed fluoroscopy for 3-dimensional research of bovine distal limb kinematics
}

\author{
M. Weiss, E. Reich, S. Grund, C. K. W. Mülling, and S. M. Geiger ${ }^{1}$ \\ Institute of Veterinary Anatomy, Histology and Embryology, Faculty of Veterinary Medicine, Leipzig University, An den Tierkliniken 43, \\ D-04103 Leipzig, Germany
}

\begin{abstract}
Lameness severely impairs cattle's locomotion, and it is among the most important threats to animal welfare, performance, and productivity in the modern dairy industry. However, insight into the pathological alterations of claw biomechanics leading to lameness and an understanding of the biomechanics behind development of claw lesions causing lameness are limited. Biplane high-speed fluoroscopic kinematography is a new approach for the analysis of skeletal motion. Biplane highspeed videos in combination with bone scans can be used for 3-dimensional (3D) animations of bones moving in 3D space. The gold standard, marker-based animation, requires implantation of radio-opaque markers into bones, which impairs the practicability for lameness research in live animals. Therefore, the purpose of this study was to evaluate the comparative accuracy of 2 noninvasive, markerless animation techniques (semiautomatic and manual) in 3D animation of the bovine distal limb. Tantalum markers were implanted into each of the distal, middle, and proximal phalanges of 5 isolated bovine distal forelimbs, and biplane high-speed $\mathrm{x}$-ray videos of each limb were recorded to capture the simulation of one step. The limbs were scanned by computed tomography to create bone models of the 6 digital bones, and 3D animation of the bones' movements were subsequently reconstructed using the marker-based, the semi-automatic, and the manual animation techniques. Manual animation translational bias and precision varied from $0.63 \pm 0.26 \mathrm{~mm}$ to $0.80 \pm 0.49 \mathrm{~mm}$, and rotational bias and precision ranged from $2.41 \pm 1.43^{\circ}$ to $6.75 \pm 4.67^{\circ}$. Semi-automatic translational values for bias and precision ranged from $1.26 \pm 1.28 \mathrm{~mm}$ to 2.75 $\pm 2.17 \mathrm{~mm}$, and rotational values varied from $3.81 \pm$ $2.78^{\circ}$ to $11.7 \pm 8.11^{\circ}$. In our study, we demonstrated the successful application of biplane high-speed fluoroscopic
\end{abstract}

Received January 7, 2017

Accepted May 28, 2017.

${ }^{1}$ Corresponding author: Monique.springer@vetmed.uni-leipzig.de kinematography to gait analysis of bovine distal limb. Using the manual animation technique, kinematics can be measured with sub-millimeter accuracy without the need for invasive marker implantation.

Key words: dairy cow, locomotion, 3D animation, bone model

\section{INTRODUCTION}

Lameness is one of the biggest threats to the dairy industry, with substantial negative effects on well-being, performance, and productivity of affected dairy cows worldwide (Warnick et al., 2001; Green et al., 2002; Bicalho et al., 2008).

Scientific analysis of the underlying causes, particularly a biomechanical understanding of claw-floor interactions, is key for successful reduction of claw tissue damage and the detection and prevention of lameness (Main et al., 2012). So far, knowledge of bovine kinematics and locomotion is based on high-speed cinematography (Meyer et al., 2007; Schmid et al., 2009; Blackie et al., 2013) or traditional radiographs (Ehlert, 2006; El Shafaey et al., 2013). But these analyses have difficulties characterizing motion of a joint that is capable of 6 degrees of freedom of movement.

Biplane high-speed fluoroscopic kinematography (HFK) is a new approach to measuring bone alignment and its changes during movement with high accuracy (Brainerd et al., 2010). This technique allows for analysis of 3-dimensional (3D) bone motion, including visualization of the distal phalanx through the surrounding horn capsule (Panagiotopoulou et al., 2016). Two x-ray generators in combination with 2 fluoroscopic image intensifiers capture high-speed video sequences (Figure 1 ). These sequences serve as templates for $3 \mathrm{D}$ bone animations of bone reconstructions and enable direct and precise measurements of bone elements in live animals during locomotion (Tashman and Anderst, 2003). So far, 3D animations have been used for motion analysis in human research (Bey et al., 2008; McDonald et al., 2010; Anderst et al., 2011; Baka et al., 2012) and in research involving different animal species such as 


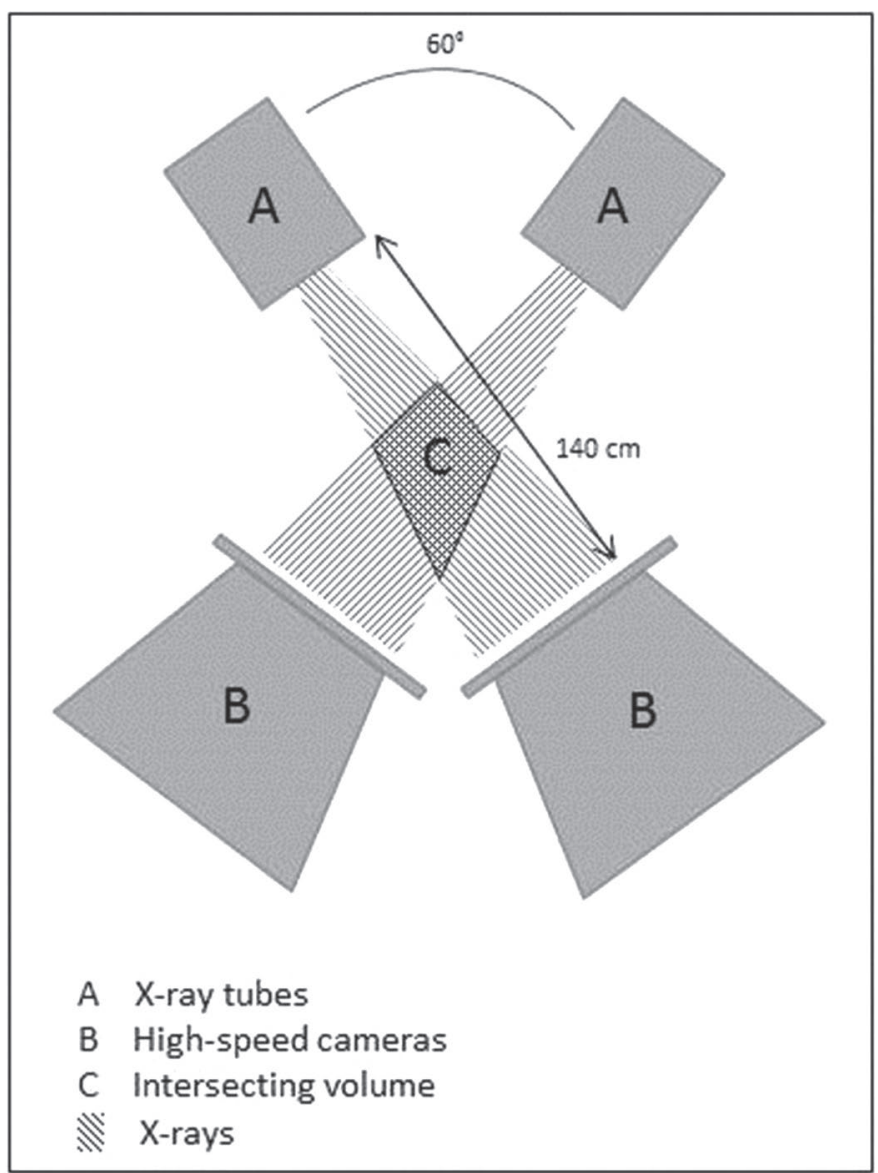

Figure 1. Setup of the biplane high-speed radiographic imaging system. The system operated with an interbeam angle of $60^{\circ}$ and an $\mathrm{x}$-ray source-to-image distance of $140 \mathrm{~cm}$. The setup was selected with regard to applicability to live dairy cows.

mini pigs (Brainerd et al., 2010), pigeons and alligators (Gatesy et al., 2010), dogs (You et al., 2001; Tashman and Anderst, 2003; Wachs et al., 2016), rabbits (Henderson et al., 2014), and horses (Panagiotopoulou et al., 2016), but not cattle.

The 3D animations can be accomplished in various ways. Marker-based registration represents the gold standard (Brainerd et al., 2010; Figure 2, top). It requires surgical implantation of at least 3 radio-opaque markers into each bone of interest to facilitate 3D animations. Although marker-based registration offers high accuracy (0.12 $\pm 0.08 \mathrm{~mm}$; Miranda et al., 2011), noninvasive markerless techniques ought to be applied to live animals for animal welfare reasons and to enable evaluation of regions that are surgically difficult to access. For noninvasive animation, 2 animation techniques are available: a semi-automatic (Figure 2, middle; Miranda et al., 2011) and a manual technique (Figure 2, bottom, Scientific Rotoscoping; Gatesy et al., 2010). Neither of these noninvasive animation techniques have been validated for the bovine distal extremity with special regard to the artiodactyle anatomy.

Because of its high resolution and high accuracy, HFK is suitable for precisely analyzing the changes of skeletal structures during movement (Brainerd et al., 2010). Applied to the bovine distal limb, HFK may allow visualization and measurement of pathological alterations of claw biomechanics. These new insights may improve the prevention of lameness; for example, by investigating claw-floor interactions on various types of flooring. They may also enable the evaluation of influences of claw-trimming methods on claw biomechanics. Digital motion as well as the influence of ground conditions may be measured precisely.

Hence, the first aim of this study was to establish the application of biplane HFK to bovine distal limb and to provide a proof of concept in describing 3D bone movement reliably and precisely. The second aim was to evaluate 2 noninvasive markerless animation techniques (semi-automatic and manual technique) with regard to future applicability in live dairy cows. We hypothesize that (1) the accuracies of the two markerless animation techniques do not differ significantly; (2) the accuracy measurements of left and right phalanges do not differ significantly; and (3) both noninvasive, markerless animation techniques can match all 6 phalanges throughout simulated steps.

\section{MATERIALS AND METHODS}

The phalanges of 5 isolated distal limbs of German Holstein dairy cows were analyzed with 3 different animation techniques with regard to bias and precision (Figure 3).

\section{Specimen Preparation}

Three left and 2 right bovine distal forelimbs, separated just below the metacarpal joint, were obtained from a local abattoir. Only limbs that were externally intact and showed no pathologies in computed tomography (CT) scans were included in this study. The digits were classified according to their proximity to the image intensifiers and cameras. The phalanges were defined as left (L; far from the camera) and right $(\mathbf{R}$; closer to the camera). Claw length was measured according to Nuss et al. (2011) (mean left claw length: $116.02 \mathrm{~mm}$; mean right claw length: $119.48 \mathrm{~mm}$ ). Proximal and middle phalanges were surgically implanted with 3 spherical tantalum markers of 1-mm diameter (X-medics Scandinavia Smba, Frederiksberg, Denmark), and distal phalanges were implanted with 4 . Markers were inserted through 2-mm venous catheters (Braun, Melsungen, Germany) into canals drilled through skin incisions into 

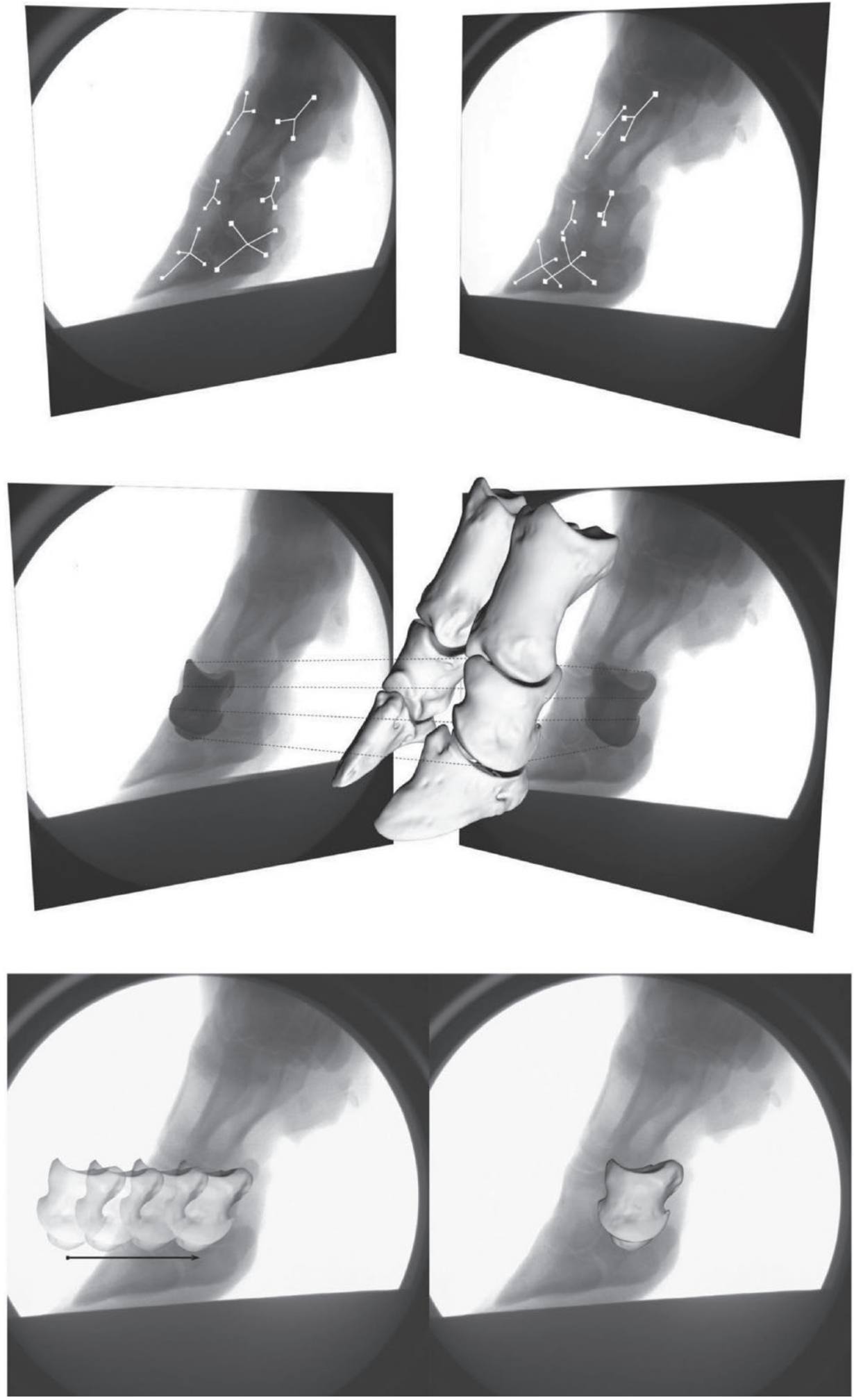

Figure 2. Marker-based animation technique. (top) View of one biplane image of the captured video sequence. Implanted bone markers are highlighted with white squares and connected with each other in corresponding bones. (middle) Example for semi-automatic animation technique. Computed tomography-based bone models (white) in front of one biplane frame of the captured video sequence. Semi-automatic matching projects (dotted lines) a digitally reconstructed radiograph (dark gray) of the left middle phalanx on the x-ray images. (bottom) Example of manual animation technique. View of one uniplane image of the captured video sequence with a 3-dimensional bone model of one middle phalanx. The bone model was manually aligned to the biplane x-ray video (left) to achieve an optimal match (right). 
the bone or into the horn capsule with a drill of 2-mm diameter and held in place with cyanoacrylate glue (TEDI GmbH \& Co. KG, Dortmund, Germany). The markers were evenly spread across each bone (Figure $4)$.

\section{Computed Tomography and Bone Models}

The CT scans of the 5 bovine limbs were performed on a multi-slice helical CT-scanner (Philips Healthcare, DA Best, the Netherlands) with a median in-plane resolution of $0.475 \mathrm{~mm}$, a slice thickness of $1 \mathrm{~mm}$, and

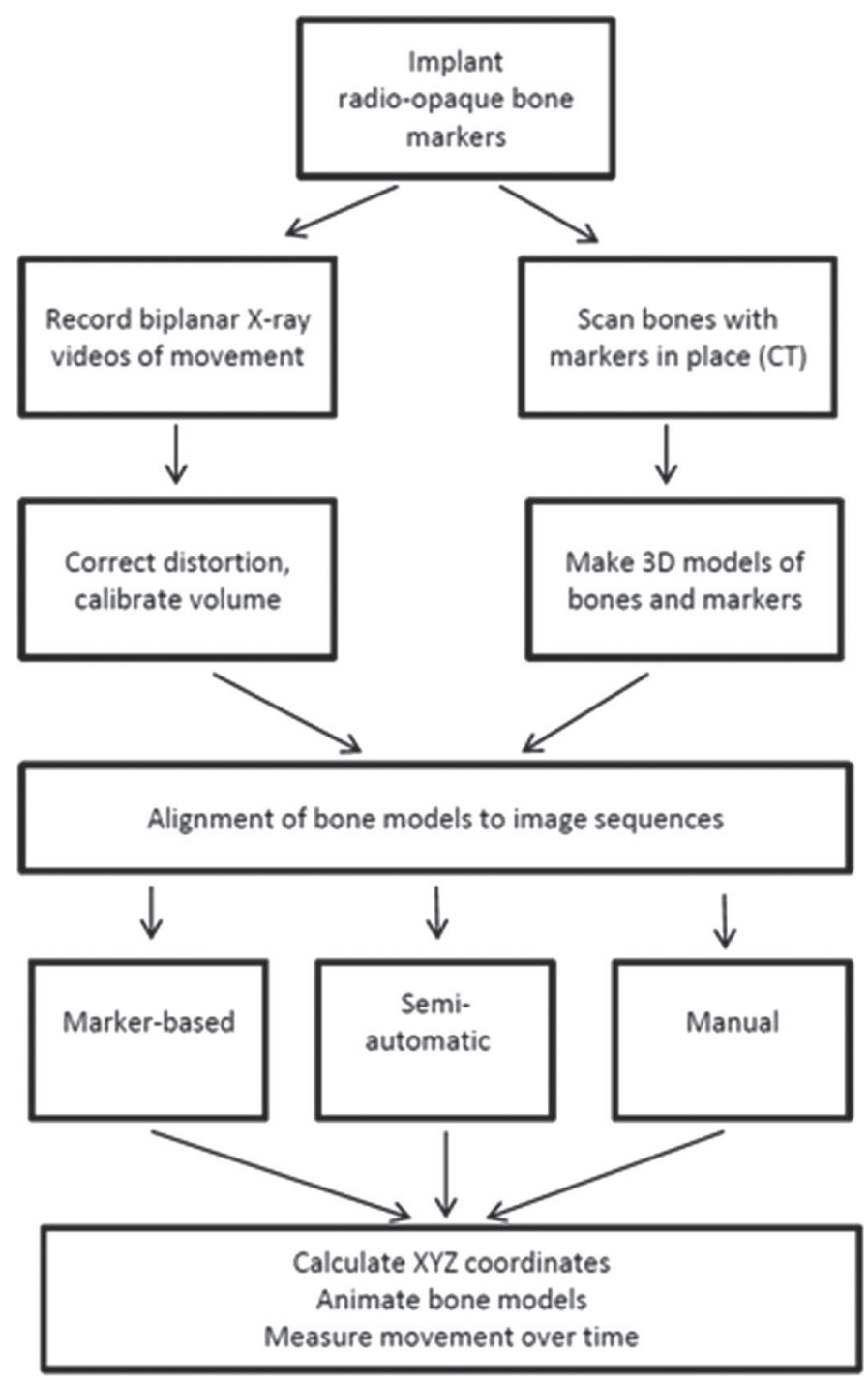

Figure 3. Overview of workflow. Upper part modified based on Brainerd et al. (2010). After implantation of radio-opaque markers, $\mathrm{x}$-ray videos and computed tomography (CT) scans were taken of 5 distal limbs, and 3-dimensional (3D) animation of the bones' movement was performed using marker-based semi-automatic and manual animation techniques.
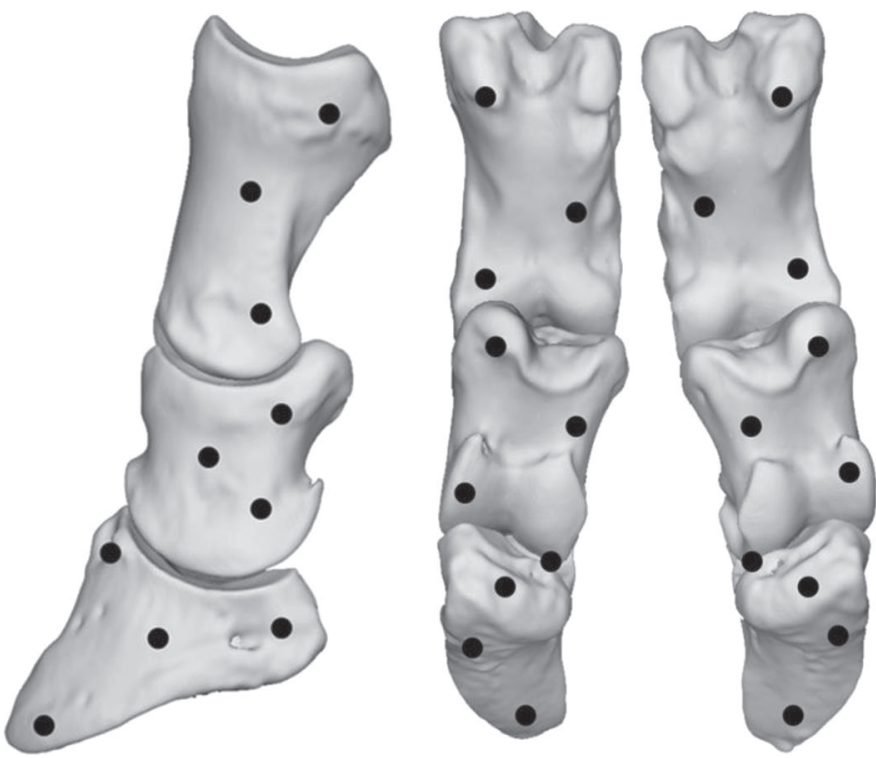

Figure 4. Example of marker positions in the 6 phalanges, lateral (left) and palmar (right) aspect. Proximal and middle phalanges were surgically implanted with 3 and distal phalanges with 4 spherical tantalum markers of 1-mm diameter.

an overlapping increment of $0.5 \mathrm{~mm}$. Model preparation was done using a sharp bone filter (Filter Type and Convolution Kernel D). The distal, middle, and proximal phalanges were manually separated from each other for bone modeling using the image processing software MeVisLab version 2.3.1 (MeVis Medical Solutions AG, Bremen, Germany). The separation allowed generation of distinct 3D surface renderings of isolated bone segments using dedicated image analysis software (VTK 3.0, Kitware Inc., Clifton Park, NY). Further enhancement, as well as inspection of the 3D models was performed if necessary (ParaView 4.1.0, Kitware Inc.). Any trace of the markers was digitally removed within the $\mathrm{CT}$ data to avoid bias in the markerless animations.

\section{Biplane High-Speed Fluoroscopy}

Two x-ray tubes (Philips Medio 65 CP-H X-Ray Generator) and 2 fluoroscopes (Philips Typ BX 3i2123) with image intensifiers and high-speed cameras (Optronis Cam Record CR600x2, Kehl, Germany) captured video sequences (Figure 1, Supplemental Video V1; https://doi.org/10.3168/jds.2017-12563) of the bones during the simulation of one step (heel strike, midstance, push off). Each limb was moved manually in the trial field of the biplane fluoroscopic setup in a step-like motion. The direction and walking velocity as they would occur in in vivo experiments were re-created. The system operated in continuous mode at 77 to $81 \mathrm{kV}$ and $100 \mathrm{~mA}$ with an interbeam angle 
of $60^{\circ}$. Video sequences with an average length of $2.4 \mathrm{~s}$ were taken at 500 frames $/ \mathrm{s}, 0.5 \mathrm{~ms}$ shutter, and a resolution of $1,024 \times 1,280$ pixels. The x-ray source-toimage distance was $1.4 \mathrm{~m}$ (Figure 1). This setup was selected with regard to applicability to live dairy cows, providing sufficient space for an in vivo walkway. Image distortion was corrected using a perforated steel sheet with a defined hole-diameter in XMALab version 1.2.17 (Brainerd et al., 2010; Knoerlein et al., 2016). Calibration of 3D space was performed with XMALab using a cube consisting of 4 layers of acrylic sheets with 64 radio-opaque markers with uniform spacing (Brainerd et al., 2010). For manual and semi-automatic animation, bone contrast on each image was enhanced using Adobe Photoshop CS2 version 9.0 Smart Sharpen filter (Adobe Systems Inc., San Jose, CA).

\section{Marker-Based 3D Animation}

Marker-based registration was done with XMALab (Figure 2, top). The software measured marker trajectories representing motion of the implanted markers over time by tracking the markers in both x-ray videos. Together with marker coordinates extracted from CT data, translations and rotations could then be calculated for every bone (Knoerlein et al., 2016). These transformations were then applied to the CT bone models in Autodesk Maya version 2014 (Autodesk Inc., San Rafael, CA) for 3D animation (Supplemental Video V2; https://doi.org/10.3168/jds.2017-12563). Additionally, standard deviations of intermarker distances were recorded.

\section{Semi-Automatic 3D Animation}

Semi-automatic tracking applies a ray-tracing algorithm to project a pair of digitally reconstructed radiographs (DRR) from the CT-based bone model onto the x-ray videos (Anderst et al., 2009; Bey et al., 2006; Figure 2, middle). After manual adjustment of the DRR to the x-ray images to obtain a good visual match for both biplane views, the software automatically completes the matching of the remaining video based on 3D bone shape and texture. The position and orientation of a bone is estimated by maximizing the match between the DRR and the biplane x-ray images. Using this technique, the $3 \mathrm{D}$ position and orientation of each phalanx were determined independently for all frames of each trial. After completion, the accordance of the DRR with every 10th x-ray image was visually verified. If a mismatch was detected, matching started again from the last frame of accordance. If the mismatch persisted, manual correction was repeated up to 3 times. If unsuccessful, the mismatch was carried through to evaluation. Semi-automatic technique data were used for 3D animations in Autodesk Maya as already described (Supplemental Video V3; https://doi .org/10.3168/jds.2017-12563).

\section{Manual 3D Animation}

For manual animation, 3D bone models were aligned manually to the biplane x-ray video in Autodesk Maya (Figure 2, bottom, Supplemental Video V4; https:// doi.org/10.3168/jds.2017-12563). The 3 bone models of every digit were organized in a hierarchy with virtual joints (Gatesy et al., 2010). In this hierarchy, the middle phalanges were placed as the "lead" bone and the distal as well as the proximal phalanges attached. So, when registering the middle phalanges' congruence with its fluoroscopic image, the other 2 bone models followed, resulting in pre-alignment. Once the middle phalanx was positioned correctly, the proximal and the distal phalanges could be matched accurately. The intervals between matched frames were continuously reduced to every eighth frame of the video sequence.

\section{Analysis of 3D Animation}

To compare the 3 different tracking techniques in terms of $3 \mathrm{D}$ bone movement, coordinate systems were applied to the bones. Each bone's center point served as its origin, while the bones' axes of inertia formed the coordinate system's axes (Crisco and McGovern, 1998).

For the evaluation of the 2 markerless animation techniques, 5 bovine distal forelimbs were used. The data of corresponding bones (left proximal phalanges, right proximal phalanges, left middle phalanges, right middle phalanges, left distal phalanges, right distal phalanges) of all 5 legs were pooled and analyzed together. Only image sequences in which all 6 bones were visible at the same time and in which all 3 tracking techniques (both markerless techniques as well as the marker-based technique) accomplished a match were analyzed. For each bone, a different number of frames was evaluated (left proximal phalanges: 3,009 ; right proximal phalanges: 2,670; left middle phalanges: 2,816 ; right middle phalanges: 2,642; left distal phalanges: 1,977; right distal phalanges: 1,033).

For further statistical analysis, 2 right distal phalanges (3 and 5) were removed. During semi-automatic matching of the right distal phalanges of claw 3 , the program stopped immediately after starting and achieved no match between the DRR and the x-ray image. For the right distal phalanges of claw 5, the semi-automatic technique worked successfully for one video. On the other camera, the DRR were arranged between the left and right distal phalanges (Figure 5). 
To evaluate the accuracy of semi-automatic and manual animation, the translational and rotational data were analyzed in relation to marker-based registration. The translational accuracy was defined as the Euclidean distance between a bone's center point tracked with the semi-automatic and marker-based technique or the manual and marker-based technique in one frame. Euclidean differences were defined as

$$
d(\mathbf{N I}, \mathbf{M B})=\sqrt{\left(x_{\mathrm{NI}}-x_{\mathrm{MB}}\right)^{2}+\left(y_{\mathrm{NI}}-y_{\mathrm{MB}}\right)^{2}+\left(z_{\mathrm{NI}}-z_{\mathrm{MB}}\right)^{2}},
$$

where NI was the $3 \mathrm{D}$ vector of the markerless tracking method and $\mathrm{MB}$ the vector of the marker-based tracking method. Accordingly, rotational accuracy was calculated using the same equation. Changes of the $\mathrm{x}$ angle represented motion in the frontal plane, y-angle in the sagittal plane, and z-angle in the transverse plane. Accuracy of manual and semi-automatic techniques was quantified in terms of bias and precision (Tashman and Anderst, 2003). Bias was defined as the average Euclidean difference of one bone type (left proximal phalanges, right proximal phalanges, left middle phalanges, right middle phalanges, left distal phalanges, right distal phalanges) over the entire image sequences of all legs in the semi-automatic and marker-based or the manual and marker-based technique. Precision was the standard deviation of the bias. All values were assessed unfiltered and filtered at $20 \mathrm{~Hz}$ using a fifth-order, low-pass Butterworth filter. For both noninvasive animation techniques, the distribution and dimension of translational and rotational tracking errors over the different phalanges are illustrated in Figures 6 and 7 with violin plots including box plots with medians, 25th and 75th percentiles, and whiskers that extend to \pm 1.5 interquartile range. Additionally, mean values of standard deviations of intermarker distances per bone

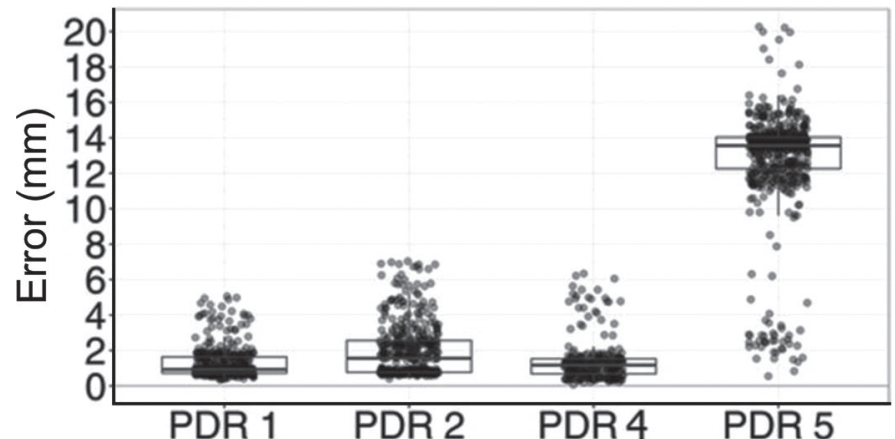

Figure 5. Distribution of semi-automatic tracking position errors of right pedal bones (PDR); PDR 5 shows at least a $12.4 \mathrm{~mm}$ larger median error than the other right phalanges. The PDR of claw 3 could not be matched with the semi-automatic tracking technique and is therefore missing from the figure. The band inside the box plot visualizes the median, bottom, and top of the box for the first and third quartiles. Whiskers in box plots extend to \pm 1.5 interquartile range. were evaluated as a measure of marker-tracking accuracy (Brainerd et al., 2010).

Statistical analysis of the data was accomplished with $\mathrm{R}$ version 3.2.4 (The R Foundation for Statistical Computing, Vienna, Austria). The Shapiro-Wilk normality test was used to determine whether values followed a normal distribution. Noninvasive animation technique values were compared with marker-based tracking using Wilcoxon's rank sum test for one sample, $P<0.01$. Comparisons of manual animation values with semiautomatic technique values and left with right phalanges were performed using the Mann-Whitney U-test, $P$ $<0.01$.

\section{RESULTS}

We successfully applied HFK to the bovine distal limb. We performed 3D animations of the proximal, middle, and distal phalanges of all 5 distal limbs with marker-based and 2 markerless animation techniques (semi-automatic and manual; Figure 2). We had to exclude 2 right distal phalanges from statistical analysis. The semi-automatic technique achieved no match between the DRR of the right distal phalanx of claw 3 and the x-ray image. The semi-automatic technique's misalignment of the right distal phalanx of claw 5 resulted in a median deviation of its translational values of at least $12.4 \mathrm{~mm}$ compared with the other right distal phalanges (Figure 5), and it was therefore excluded.

As a measure of marker-tracking accuracy, we recorded standard deviations of intermarker distances of $0.04 \mathrm{~mm}$ ( $\min 0.005 \mathrm{~mm}$; $\max 0.14 \mathrm{~mm}$ ) for all bones and limbs.

In translational and rotational analysis, low values indicate a high tracking accuracy and high values a low tracking accuracy. The Shapiro-Wilk normality test resulted in 0.00 for every group, showing that values did not follow a normal distribution.

\section{Translation}

The distribution and amount of unfiltered translational tracking errors of manual and semi-automatic techniques are shown in Figure 6 with violin plots in combination with box plots. Unfiltered translational bias and precision as well as medians and 95\% confidence intervals (CI) for semi-automatic and manual animation are given in Table 1. Unfiltered manual animation bias and precision varied from $0.63 \pm 0.26 \mathrm{~mm}$ (left distal phalanges) to $0.80 \pm 0.49 \mathrm{~mm}$ (right distal phalanges). Manual animations' lowest medians occurred with $0.58 \mathrm{~mm}$ for right middle phalanges and the highest with $0.71 \mathrm{~mm}$ for right distal phalanges. Unfiltered semi-automatic technique bias and preci- 
sion ranged from $1.26 \pm 1.28 \mathrm{~mm}$ (right middle phalanges) to $2.75 \pm 2.17 \mathrm{~mm}$ (left distal phalanges). Semi-automatic techniques' lowest medians arose with $0.76 \mathrm{~mm}$ for right middle phalanges and the highest with $2.09 \mathrm{~mm}$ for left distal phalanges. For both noninvasive animation techniques, medians were lower in comparison with bias.

In a comparison of the lowest 95\% CI value for the semi-automatic technique with the highest $95 \%$ CI value for the manual animation (mindiff) and vice versa (maxdiff), the magnitude of the difference between both markerless techniques ranged from 1.5 up to 4.1 in favor of manual animation. On average, manual animation showed medians that were 2.1 times lower than those of the semi-automatic technique. Manual animation showed continuously lower values for translation than the semi-automatic technique for all bones $(P<$ $0.01)$. Noninvasive animation methods were different in translation from the marker-based method $(P<0.01)$.

In a comparison of the values of left and right phalanges, both markerless techniques showed differences in translation between each left and right phalanx $(P$
$<0.01)$. For the semi-automatic animation technique, higher errors (median and 95\% CI maximum, Table 1) occurred in left phalanges compared with corresponding right phalanges across all bones. The shape of the violin plots of the semi-automatic technique in Figure 6 also suggests a higher probability of higher errors in left phalanges. The manual method did not show this consistent distribution. Higher translational values were generated for right proximal and distal phalanges, whereas values for right middle phalanges were lower than the corresponding left side (Table 1 and Figure 6).

For detailed information of bias and precision and the median distribution in the $\mathrm{x}-, \mathrm{y}-$, and $\mathrm{z}$-axes see Supplemental Table S1 (https://doi.org/10.3168/jds .2017-12563). For detailed information of filtered data, see Supplemental Table S2 and S3 (https://doi.org/10 $.3168 /$ jds.2017-12563).

\section{Rotation}

Figure 7 shows the distribution and dimensions of unfiltered rotational tracking errors of manual and

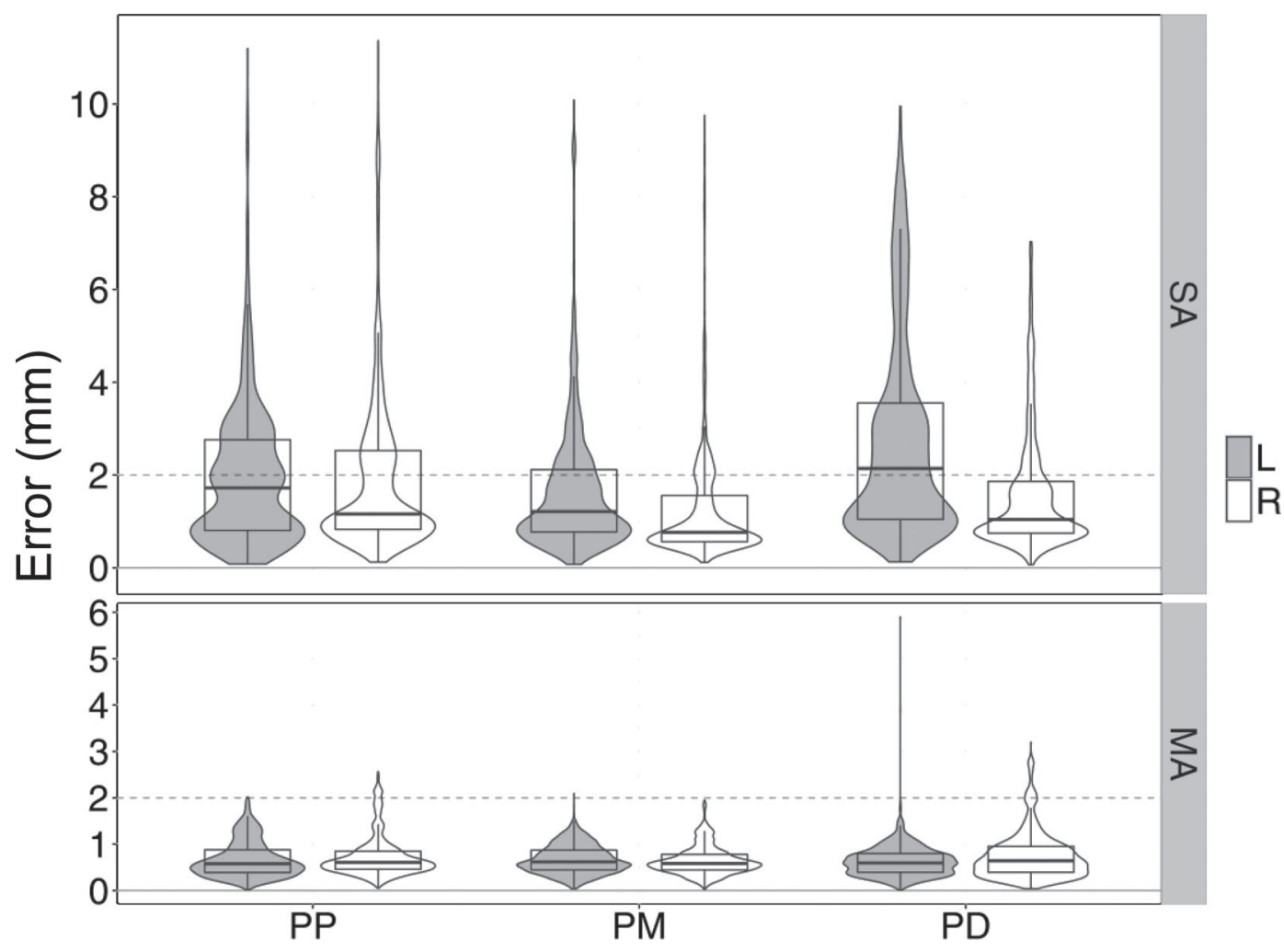

Figure 6. Distribution and dimensions of translational tracking errors of semi-automatic (SA) and manual (MA) animation techniques visualized by violin plots with boxplots for proximal (PP), middle (PM), and distal (PD) left (L) and right (R) phalanges. The band inside the box plot visualizes the median, bottom, and top of the box for the first and third quartiles. Whiskers in boxplots extend to \pm 1.5 interquartile range. The violin plots' maximum width was the same for every bone to permit visualizing the relative error distribution among bones. For example, the SA technique's violin plots' maximum width for proximal phalanges is $0.7 \mathrm{~mm}$, which means that the greatest number of errors is located at $0.7 \mathrm{~mm}$. 
Table 1. Unfiltered translational bias and precision values with median and $95 \%$ CI for the different bone types of both markerless animation techniques

\begin{tabular}{|c|c|c|c|c|c|}
\hline \multirow[b]{2}{*}{ Bone $^{1}$} & \multirow[b]{2}{*}{$\mathrm{n}^{2}$} & \multirow[b]{2}{*}{ Method $^{3}$} & \multicolumn{3}{|c|}{ Translation } \\
\hline & & & Bias and precision & Median & $95 \% \mathrm{CI}$ \\
\hline \multirow[t]{2}{*}{ PPL } & 3,009 & $\mathrm{MA}^{\mathrm{a}}$ & $0.69 \pm 0.39$ & $0.59^{\mathrm{c} *}$ & $0.63-0.66$ \\
\hline & & $\mathrm{SA}^{\mathrm{b}}$ & $1.96 \pm 1.54$ & $1.72^{\mathrm{c} *}$ & $1.74-1.84$ \\
\hline \multirow[t]{2}{*}{ PPR } & 2,670 & $\mathrm{MA}^{\mathrm{a}}$ & $0.73 \pm 0.45$ & $0.60^{\mathrm{d} *}$ & $0.62-0.65$ \\
\hline & & $\mathrm{SA}^{\mathrm{b}}$ & $1.91 \pm 1.73$ & $1.16^{\mathrm{d} *}$ & $1.57-1.67$ \\
\hline \multirow[t]{2}{*}{ PML } & 2,816 & $\mathrm{MA}^{\mathrm{a}}$ & $0.70 \pm 0.30$ & $0.65^{\mathrm{c} *}$ & $0.67-0.70$ \\
\hline & & $\mathrm{SA}^{\mathrm{b}}$ & $1.67 \pm 1.43$ & $1.21^{\mathrm{c} *}$ & $1.38-1.47$ \\
\hline \multirow[t]{2}{*}{ PMR } & 2,642 & $\mathrm{MA}^{\mathrm{a}}$ & $0.64 \pm 0.29$ & $0.58^{\mathrm{d} *}$ & $0.59-0.62$ \\
\hline & & $\mathrm{SA}^{\mathrm{b}}$ & $1.26 \pm 1.28$ & $0.76^{\mathrm{d} *}$ & $0.93-1.03$ \\
\hline \multirow[t]{2}{*}{ PDL } & 1,977 & $\mathrm{MA}^{\mathrm{a}}$ & $0.63 \pm 0.26$ & $0.63^{\mathrm{c} *}$ & $0.61-0.64$ \\
\hline & & $\mathrm{SA}^{\mathrm{b}}$ & $2.75 \pm 2.17$ & $2.09^{\mathrm{c} *}$ & $2.27-2.47$ \\
\hline \multirow[t]{2}{*}{ PDR } & 1,033 & $\mathrm{MA}^{\mathrm{a}}$ & $0.80 \pm 0.49$ & $0.71^{\mathrm{d} *}$ & $0.71-0.77$ \\
\hline & & $\mathrm{SA}^{\mathrm{b}}$ & $1.50 \pm 1.30$ & $0.95^{\mathrm{d} *}$ & $1.12-1.23$ \\
\hline
\end{tabular}

$\overline{\mathrm{a}, \mathrm{b}}$ Data of MA or SA for proximal, middle, and distal phalanges with dissimilar superscripts within a column differ $(P<0.01)$.

${ }^{\mathrm{c}, \mathrm{d}}$ Data of left and right proximal, middle, and distal phalanges with dissimilar superscripts within a column differ $(P<0.01)$.

${ }^{1} \mathrm{PPL}=$ left proximal phalanges; $\mathrm{PPR}=$ right proximal phalanges; $\mathrm{PML}=$ left middle phalanges; $\mathrm{PMR}=$ right middle phalanges; $\mathrm{PDL}=$ left distal phalanges; $\mathrm{PDR}=$ right distal phalanges.

${ }^{2} \mathrm{n}=$ image sequence length statistically analyzed for each bone type.

${ }^{3} \mathrm{MA}=$ manual animation technique; $\mathrm{SA}=$ semi-automatic animation technique.

*Data differ from marker-based animation $(P<0.01)$.

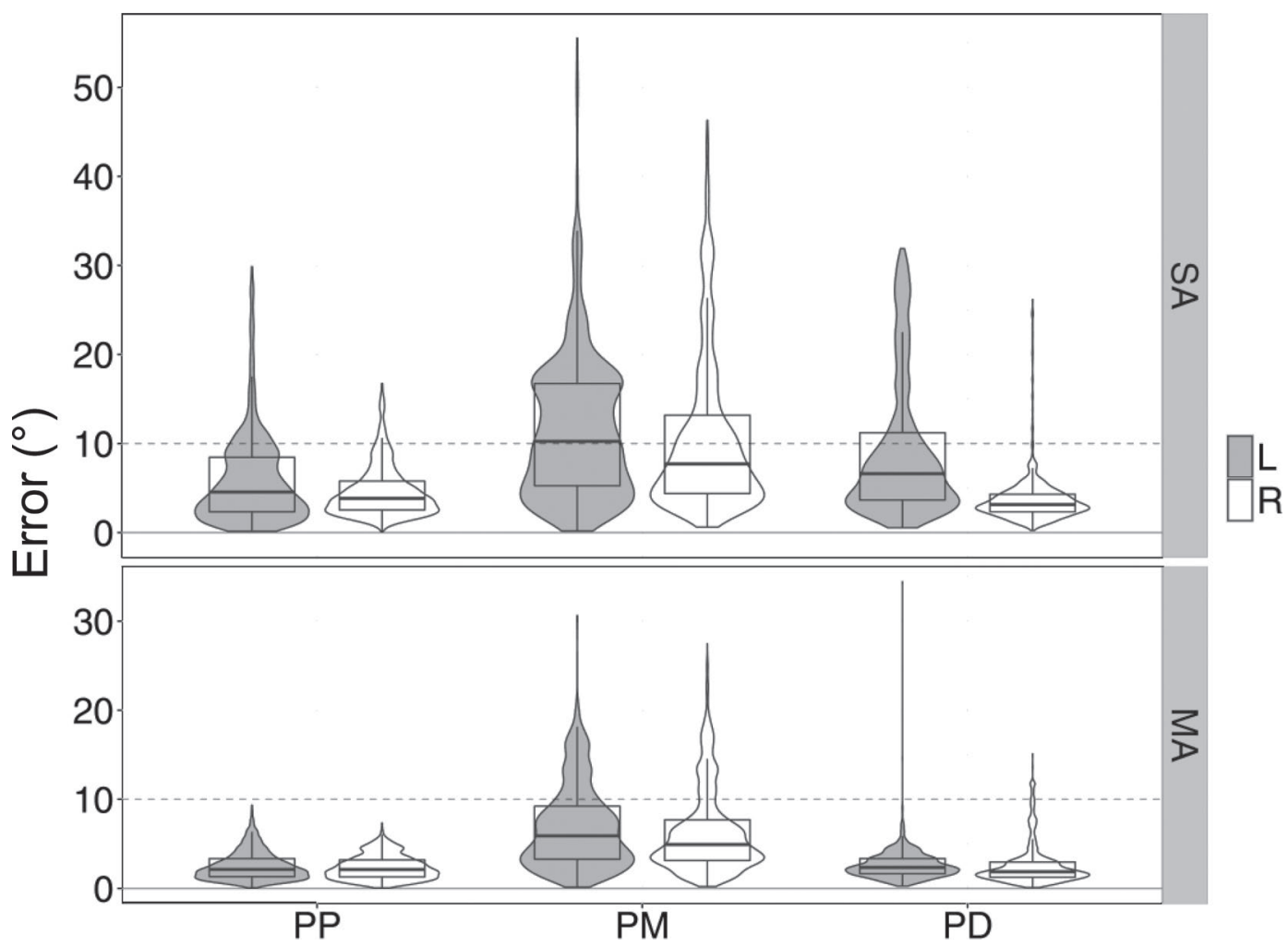

Figure 7. Distribution and dimensions of rotational tracking errors of semi-automatic (SA) and manual (MA) animation techniques visualized by violin plots with box plots for proximal (PP), middle (PM), and distal (PD) left (L) and right (R) phalanges. The band inside the box plot visualizes the median, bottom, and top of the box for the first and third quartiles. Whiskers in boxplots extend to \pm 1.5 interquartile range. The violin plots' maximum width was the same for every bone to permit visualizing the relative error distribution among bones. For example, the SA technique's violin plots' maximum width for proximal phalanges is $2.5^{\circ}$, which means that the greatest number of errors is located at $2.5^{\circ}$. 
semi-automatic animation. Table 2 shows unfiltered rotational bias and precision as well as medians and $95 \%$ CI for semi-automatic and manual animation. Unfiltered manual animation bias and precision ranged from $2.41 \pm 1.43^{\circ}$ (right proximal phalanges) to $6.75 \pm 4.67^{\circ}$ (left middle phalanges). Manual animation's lowest medians occurred with $2.09^{\circ}$ for right proximal phalanges and the highest with $5.63^{\circ}$ for the left middle phalanges. Unfiltered semi-automatic technique bias and precision varied from $3.81 \pm 2.78^{\circ}$ (right distal phalanges) to $11.7 \pm 8.11^{\circ}$ (left middle phalanges). Semi-automatic techniques' lowest medians were $3.26^{\circ}$ for right distal phalanges and the highest were $10.25^{\circ}$ for left middle phalanges. Medians were lower in comparison with bias for both noninvasive animation techniques.

In a comparison of semi-automatic techniques' lowest 95\% CI value with manual animation's highest 95\% CI value (mindiff) and vice versa (maxdiff), the accuracy between both markerless techniques ranged from 1.3 to 3.4 times in favor of manual animation. On average, manual animation showed 2.0 times higher rotational accuracy than semi-automatic animation. Manual animation showed consistently lower values for rotation than semi-automatic animation $(P<0.01)$. Rotational accuracy was lower than translational accuracy for all phalanges in both noninvasive animation techniques. Rotations in both markerless tracking techniques were different from the marker-based method $(P<0.01)$.

The analysis of corresponding bones' tracking accuracy revealed that manual animation showed a dif- ference in rotational accuracy between corresponding middle phalanges $(P<0.01)$, but not for proximal phalanges $(P=0.87)$ or distal phalanges $(P=0.04)$. Semi-automatic technique showed differences in rotation between the left and right side for middle phalanges and distal phalanges $(P<0.01)$, but not for proximal phalanges $(P=0.03)$. In accordance with the results for translational values for the semi-automatic animation technique, higher rotational errors occurred in left phalanges compared with corresponding right phalanges across all bones. Manual techniques' lowest medians were also found in left phalanges compared with corresponding right phalanges across all bones (Table 2 and Figure 7 ).

For detailed information of bias and precision and the median distribution in the $\mathrm{x}-, \mathrm{y}-$, and $\mathrm{z}$-angles see Supplemental Table S4 (https://doi.org/10.3168/jds .2017-12563). For detailed information of filtered data, see Supplemental Table S5 and S6 (https://doi.org/10 $.3168 /$ jds.2017-12563).

\section{DISCUSSION}

In this study, we successfully applied biplane highspeed fluoroscopy to the bovine distal limb and evaluated 2 markerless animation techniques (semi-automatic and manual) for noninvasive kinematic measurements of bovine phalanges. To determine bias and precision as well as medians, we used marker-based tracking as the gold standard. As a measure of marker-tracking accu-

Table 2. Unfiltered rotational bias and precision values with median and $95 \%$ CI for the different bone types of both markerless animation techniques

\begin{tabular}{|c|c|c|c|c|c|}
\hline \multirow[b]{2}{*}{ Bone $^{1}$} & \multirow[b]{2}{*}{$n^{2}$} & \multirow[b]{2}{*}{ Method $^{3}$} & \multicolumn{3}{|c|}{ Rotation } \\
\hline & & & Bias and precision & Median & $95 \% \mathrm{CI}$ \\
\hline \multirow[t]{2}{*}{ PPL } & \multirow[t]{2}{*}{3,009} & $\mathrm{MA}^{\mathrm{a}}$ & $2.57 \pm 1.66$ & $2.11^{\mathrm{c} *}$ & $2.33-2.46$ \\
\hline & & $\mathrm{SA}^{\mathrm{b}}$ & $5.93 \pm 5.05$ & $4.56^{\mathrm{c} *}$ & $5.13-5.45$ \\
\hline \multirow[t]{2}{*}{ PPR } & \multirow[t]{2}{*}{2,670} & $\mathrm{MA}^{\mathrm{a}}$ & $2.41 \pm 1.43$ & $2.09^{\mathrm{c} *}$ & $2.26-2.39$ \\
\hline & & $\mathrm{SA}^{\mathrm{b}}$ & $4.62 \pm 2.86$ & $3.84^{\mathrm{c} *}$ & $4.09-4.30$ \\
\hline \multirow[t]{2}{*}{ PML } & \multirow[t]{2}{*}{2,816} & $\mathrm{MA}^{\mathrm{a}}$ & $6.75 \pm 4.67$ & $5.63^{\mathrm{c} *}$ & $5.99-6.34$ \\
\hline & & $\mathrm{SA}^{\mathrm{b}}$ & $11.70 \pm 8.11$ & $10.25^{\mathrm{c} *}$ & $10.71-11.24$ \\
\hline \multirow[t]{2}{*}{ PMR } & \multirow[t]{2}{*}{2,642} & $\mathrm{MA}^{\mathrm{a}}$ & $6.36 \pm 4.47$ & $5.12^{\mathrm{d} *}$ & $5.54-5.92$ \\
\hline & & $\mathrm{SA}^{\mathrm{b}}$ & $10.60 \pm 8.72$ & $7.71^{\mathrm{d} *}$ & $8.55-9.19$ \\
\hline \multirow[t]{2}{*}{ PDL } & \multirow[t]{2}{*}{1,977} & $\mathrm{MA}^{\mathrm{a}}$ & $2.56 \pm 1.39$ & $2.26^{\mathrm{c} *}$ & $2.40-2.53$ \\
\hline & & $\mathrm{SA}^{\mathrm{b}}$ & $9.43 \pm 7.86$ & $6.74^{\mathrm{c} *}$ & $7.38-8.12$ \\
\hline \multirow[t]{2}{*}{ PDR } & \multirow[t]{2}{*}{1,033} & $\mathrm{MA}^{\mathrm{a}}$ & $2.76 \pm 2.23$ & $2.10^{\mathrm{c} *}$ & $2.27-2.52$ \\
\hline & & $\mathrm{SA}^{\mathrm{b}}$ & $3.81 \pm 2.78$ & $3.26^{\mathrm{d} *}$ & $3.32-3.52$ \\
\hline \multicolumn{6}{|c|}{$\begin{array}{l}\mathrm{a}, \mathrm{b} \\
\text { Data of MA or SA for proximal, middle, and distal phalanges with dissimilar superscripts within a column } \\
\text { differ }(P<0.01) \text {. }\end{array}$} \\
\hline \multicolumn{6}{|c|}{$\begin{array}{l}{ }^{c, d} \text { Data of left and right proximal, middle, and distal phalanges with dissimilar superscripts within a column } \\
\text { differ }(P<0.01) .\end{array}$} \\
\hline \multirow{2}{*}{\multicolumn{6}{|c|}{$\begin{array}{l}{ }^{1} \mathrm{PPL}=\text { left proximal phalanges; } \mathrm{PPR}=\text { right proximal phalanges; } \mathrm{PML}=\text { left middle phalanges; } \mathrm{PMR}=\text { right } \\
\text { middle phalanges; } \mathrm{PDL}=\text { left distal phalanges; } \mathrm{PDR}=\text { right distal phalanges. } \\
{ }^{2} \mathrm{n}=\text { image sequence length statistically analyzed for each bone type. }\end{array}$}} \\
\hline & & & & & \\
\hline \multicolumn{6}{|c|}{${ }^{3} \mathrm{MA}=$ manual animation technique; $\mathrm{SA}=$ semi-automatic animation technique. } \\
\hline
\end{tabular}


racy, standard deviations of intermarker distances were recorded. We observed a mean of standard deviations of unfiltered intermarker distances lower than the mean values of previous reports: 0.064 and $0.072 \mathrm{~mm}$ (filtered) (Tashman and Anderst, 2003); $0.089 \mathrm{~mm}$ (unfiltered) (Brainerd et al., 2010); $0.09 \mathrm{~mm}$ (Anderst et al., 2011); and $0.062 \pm 0.010 \mathrm{~mm}$ (Knoerlein et al., 2016). This finding is indicative of high marker-tracking accuracy and a rigid connection between markers and bones. It demonstrates that marker positions within bones did not change during limb motion. Marker-based tracking therefore provided a precise reference for the evaluation of both noninvasive animation techniques.

The manual simulation of the steps in this study was created to visually resemble in vivo steps in radiographic recordings. Care was taken to meet general radiographically visible criteria of a step (i.e., heel strike, midstance, push off) with velocity representative of a walking cow. In addition, superimposition of the phalanges was accurately re-created according to the expected situation in in vivo experiments. However, although vertical and accelerating-braking forces have been generated, they were not comparable to the in vivo situation. Because it was not technically possible to realistically simulate both appearance and forces of a bovine step at the same time, the parameter that was more likely to affect the results of this study (i.e., appearance) was prioritized to meet the required criteria. Therefore, this study provides a proof of concept for the application of noninvasive tracking techniques to the bovine distal limb. Future studies will have to determine their accuracy under real load-bearing conditions and when load bearing shifts during lameness.

For the evaluation of the 2 noninvasive animation methods, we used 5 bovine distal forelimbs (3 left and 2 right limbs). Limbs were not divided into left and right because no morphometrical differences exist between left and right phalanges (Ocal et al., 2004). Besides, for the results of $3 \mathrm{D}$ bone model animation, superimposition of the 2 digits in the biplane $\mathrm{x}$-ray videos is very important. Because of this superimposition, validation of HFK if the phalanges were farther or closer to the cameras was of major interest. Therefore, we decided to classify the digits as left (far from the camera) and right (closer to the camera). For statistical analysis we had to exclude 2 right distal phalanges (3 and 5 ). For the right distal phalanx of claw 3 the semi-automatic technique was not able to accomplish a match between the DRR and the x-ray image at all. For the right distal phalanx of claw 5 the semi-automatic technique arranged the DRR between left and right distal phalanges on one camera, whereas the other view worked satisfactorily. In oblique biplane recording of artiodactyle phalanges, one of the corresponding bones is the anterior and the other is the posterior in one view; in the second view, the perspectives are reversed. The misalignment of the right distal phalanx of claw 5 occurred in the view in which it was the posterior one and therefore more heavily influenced by superimposition, which may explain the semi-automatic technique's difficulties in matching the DRR with the x-ray image. The reason why only 2 of the 5 right distal phalanges were affected by misalignment may be due to the indistinct bone shape of these 2 right distal phalanges. Consequently, semiautomatic technique may have encountered problems with finding and differentiating bone contours. The removal of 2 of the right distal phalanges for statistical computing reduced the analyzed number of frames to 1,033 , which was relatively small compared with the other phalanges, but the number was sufficient for statistical analysis.

For manual animation, the bone models were placed in a hierarchy with virtual joints (Gatesy et al., 2010). This attachment in an articular chain provided the benefit of transferring the rotations and translations of the "lead bone" (middle phalanges) to all bone models rather than rotoscoping each bone entirely independently. The resulting pre-alignment of the attached bone models saved time, but only if the lead bone was matched with high accuracy itself. We chose the middle phalanges as lead bones because of their position within the central beam. Although low values for middle phalanges translation were recorded, the high values for rotation (up to 2.7 times higher than the other phalanges) necessitate a reconsideration of the hierarchy used. These high rotational values for the middle phalanges may arise from its rotund shape and indistinct contours, which particularly occur at its distal parts due to the horn capsule. These high values may also be connected to the middle phalanges' position as the lead bone because it was the first bone model to be aligned manually to the biplane x-ray images. The bone models of the other phalanges followed according to the hierarchy and had to undergo less manipulation to be matched accurately. The time-saving benefit of a hierarchy is a great advantage, but for further studies we suggest placing the proximal phalanges at the top level. Because of their long shape and defined bone contour, the proximal phalanges' bone models could be aligned more accurately to the biplane x-ray videos, resulting in lower bias values for translation and rotation. With the proximal phalanx as the lead bone, its accurate alignment can be transferred to the middle and distal phalanges.

Although HFK is a complex laboratory-based method, it has the potential to enable new insights in claw biomechanics by direct and precise measurements of bone movement. In the future, HFK may also permit 
visualizing and measuring digital motion and allow for investigation of claw-floor interactions for various types of flooring or evaluation of the influences of claw-trimming methods. These new insights can help to improve the understanding of the development and prevention of lameness.

The size of the biplane x-ray system's field of view, which was defined by the overlapping region of the crossing x-ray beams (Figure 1), determined the extent to which skeletal motion could be measured. The intersecting volume of the 2 x-ray beams is approximately $6,000 \mathrm{~cm}^{3}$ (Brainerd et al., 2010). This limit means that the full range of one step of a walking cow cannot be captured in a single take. For the development of lameness, standing and walking on hard flooring are main risk factors (Bergsten, 2001; Cook and Nordlund, 2009; Burow et al., 2014). Therefore, we focused on heel strike, midstance, and push off of the limb. We chose a setup with an interbeam angle of $60^{\circ}$ and a source-to-image distance of $1.4 \mathrm{~m}$. This arrangement allowed us to keep a walkway of more than 1-m width clear of equipment for future in vivo application. However, an increased angle of $90^{\circ}$ may improve the spatial resolution, and a different arrangement of x-ray tubes and cameras allowing for a clear distinction between the phalanges will probably have a positive effect on the accuracy of noninvasive animation techniques. The selected setup enables the direct applicability of this study to future studies on live animals, but future hardware developments will have to address this issue.

This study presents the first application of HFK to the bovine distal limb. The 2 markerless animation techniques (semi-automatic and manual) for noninvasive kinematic measurements of bovine phalanges were evaluated in relation to marker-based registration. Marker-based registration is the gold standard, with a methodological error of $0.12 \pm 0.08 \mathrm{~mm}$ and $0.09 \pm$ $0.08^{\circ}$ (Miranda et al., 2011). This error has to be added to the translational and rotational bias and precision of both the manual and semi-automatic measurements. The comparison of the reported results to previous studies is difficult because the previous investigations pertained to entirely different anatomical structures in other species. To our knowledge, only 1 reference exists for the accuracy of the manual technique validating markerless animation techniques in equines (Geiger et al., 2017). We found translational accuracy to be in accordance with data of the equine study for proximal and middle phalanges, whereas the distal phalanges showed lower accuracy. Rotational accuracy median values were up to 1.6 times higher for all phalanges. For the semi-automatic technique, we observed translational and rotational bias values and precision of at least $0.45 \mathrm{~mm}$ and $2.82^{\circ}$ to be higher than those pre- viously published when using a shape-based tracking technique: $0.0 \pm 0.56 \mathrm{~mm}$ and $0.0 \pm 0.61^{\circ}$ (McDonald et al., 2010); $0.1 \pm 0.59 \mathrm{~mm}, 0.01 \pm 0.65^{\circ}$ (Giphart et al., 2012 ); and 0.48 to $0.81 \mathrm{~mm}, 0.69$ to $0.99^{\circ}$ (Baka et al., 2014). The paired digits might explain the differences in translational and rotational bias and precision across semi-automatic and manual animation techniques. The superimposition and the resulting overlapping bone contours impaired semi-automatic technique's matching of the DRR to the x-ray images. Other factors may be irregular bone shape or texture that compromised the semi-automatic technique's performance of distinguishing between bone contours. Additionally, the horn capsule superimposes the lower part of the middle and the entire distal phalanges. In relation, impaired semiautomatic matching became particularly apparent in the left distal phalanges, resulting in high bias values.

The accuracies of both markerless animation techniques do differ $(P<0.01)$. Therefore, our first hypothesis stating that the accuracies of both markerless animation techniques do not differ significantly has to be rejected. Overall, manual animation showed higher translational and rotational tracking accuracy than semi-automatic animation $(P<0.01)$, which proves manual animation to be the preferable markerless tracking technique for bovine distal limbs. The differences in accuracy between both markerless techniques are directly visible in the created animations. For example, in the semi-automatic 3D animation considerable translational and rotational movements of the middle phalanges in relation to the distal and proximal phalanges are visible in Supplemental Video V3. In connection with the presented results, these movements are even more pronounced in the left bones, which are the ones farther from the camera. Because of the lower translational and rotational errors of the manual method, jerky movements of the individual bones in the manual animation (Supplemental Video V4) are less prominent compared with the ones in the semi-automatic animation. The animations in Supplemental Videos V1 to V4 (https://doi.org/10.3168/jds.2017-12563) all depict the same motion. Supplemental Video V1 shows the captured biplane high-speed fluoroscopic recordings, and V2 shows the marker-based animation of the bones in the biplane videos. Supplemental Video V3 depicts the semi-automatic animation of those bones and V4 the manual animation of the same bones. Supplemental Video V2 represents the gold standard. All movements that do not occur in V2 but are visible in V3 and V4 are therefore attributed to differences introduced by the noninvasive techniques.

We examined the tracking errors of corresponding bones to investigate if the accuracies of left and right phalanges differed. Both noninvasive animation tech- 
niques showed a difference in all corresponding bones for translational accuracy $(P<0.01)$. For rotational accuracy, we found only a difference for middle phalanges and semi-automatic technique's matching of distal phalanges $(P<0.01)$. Semi-automatic matching showed consistently better results for right phalanges, while manual animation values revealed no side preference. This result further supports use of the manual over the semi-automatic technique. Our second hypothesis stating that the accuracies of left and right phalanges do not differ significantly has to be partially rejected. The software accomplishes the matching between the DRR and the biplane x-ray images based on 3D bone shape and texture. The side preference of semi-automatic tracking may be explained with differences in image quality where one bone side possibly had distinctive contours, which increased detection by the software. The superimposition of bone structures and the resulting reduction in bone contour, which likely impaired semi-automatic matching, are the same for manual animation, but animator skill and anatomical knowledge could increase the accuracy of detail recognition. For both noninvasive animation techniques, bone contrast in each image was enhanced using an image sharpening filter. For further increase of animation accuracy, each image may be enhanced individually. The image contrast of the posterior and therefore the superimposed bone should be optimized for each camera independently from the other phalanges to improve their visibility.

The amount of time needed to create the animations is an important factor. Even when the bones were placed in a hierarchy with virtual joints, each bone had to be aligned individually for proper accordance of bone models with the x-ray image. Depending on the video sequence, manual animation of one distal limb took approximately 80 to $120 \mathrm{~h}$. Semi-automatic animation took approximately the same amount of time because of low reliability of the software, numerous mismatches, and frequent necessity of verification of DRR accordance with the x-ray image. At the same time, the semi-automatic technique produced higher translational and rotational values but, as mentioned previously, was not capable of matching all 6 phalanges. Future software developments, such as improvement of bone contour detection for semi-automatic technique or enhancement of the images, may improve results. Nevertheless, our third hypothesis stated that both noninvasive markerless animation techniques are capable of matching all 6 phalanges throughout simulated steps has to be rejected for the semi-automatic technique. In contrast, our hypothesis is confirmed for manual animation. Manual animation achieved 3D animations of every phalanx, producing results with high accuracy, which offsets the effort and the expenditure of time.
An appropriate bone hierarchy might increase manual animation's accuracy and may save time.

\section{CONCLUSIONS}

We successfully applied the method of biplane highspeed fluoroscopy to the bovine distal limb for the first time. We evaluated 2 noninvasive markerless animation techniques in a simulation model. Our results clearly show that bovine distal limb kinematics can be precisely measured with sub-millimeter accuracy without the need for invasive marker implantation. The ex vivo validation indicates that the manual animation method is the better choice. It is accurate to within 0.63 to $0.80 \mathrm{~mm}$ in translation and 2.41 to $6.75^{\circ}$ in rotation. It provides a reference for the level of accuracy achievable for studies in vivo. It may enable new insights into claw biomechanics and claw-floor interactions, a key factor in understanding the pathogenesis of lameness as well as furthering its prevention. In the future, these animations may be applied to live animals to permit visualizing and evaluating movement of the distal phalanx inside the horn capsule. This technique represents a new, innovative approach for analyzing 3D bone and joint kinematics in 6 degrees of freedom for further locomotion research to maintain the well-being, performance, and productivity of dairy cows.

\section{ACKNOWLEDGMENTS}

The project was supported by funds of the Landwirtschaftliche Rentenbank (Frankfurt am Main, Germany). The authors acknowledge Peter Boettcher (Department of Veterinary Medicine at the Freie Universitaet Berlin, Germany) and Dirk Brause, Juliane Munzel, Benjamin Oehme, and Nicole Röhrmann (Faculty of Veterinary Medicine of Leipzig University, Germany) for assisting with the collection of data and supporting figure preparation. The authors declare no competing or financial interests.

\section{REFERENCES}

Anderst, W. J., E. Baillargeon, W. F. Donaldson 3rd, J. Y. Lee, and J. D. Kang. 2011. Validation of a noninvasive technique to precisely measure in vivo three-dimensional cervical spine movement. Spine 36:E393-E400. https://doi.org/10.1097/BRS.0b013e31820b7e2f.

Anderst, W., R. Zauel, J. Bishop, E. Demps, and S. Tashman. 2009. Validation of three-dimensional model-based tibio-femoral tracking during running. Med. Eng. Phys. 31:10-16. https://doi.org/10 .1016/j.medengphy.2008.03.003.

Baka, N., M. de Bruijne, T. van Walsum, B. L. Kaptein, J. E. Giphart, M. de Schaap, B. P. F. Levieveldt, and E. Valstar. 2012. Statistical shape model-based femur kinematics from biplane fluoroscopy. IEEE Trans. Med. Imaging 31:1573-1583. https://doi.org/ 10.1109/TMI.2012.2195783.

Baka, N., B. L. Kaptein, J. E. Giphart, M. Staring, M. de Bruijne, B. P. F. Lelieveldt, and E. Valstar. 2014. Evaluation of automat- 
ed statistical shape model based knee kinematics from biplane fluoroscopy. J. Biomech. 47:122-129. https://doi.org/10.1016/j .jbiomech.2013.09.022.

Bergsten, C. 2001. Effects of conformation and management system on hoof and leg diseases and lameness in dairy cows. Vet. Clin. North Am. Food Anim. Pract. 17:1-23.

Bey, M. J., S. K. Kline, R. Zauel, T. R. Lock, and P. A. Kolowich. 2008. Measuring dynamic in vivo glenohumeral joint kinematics: Technique and preliminary results. J. Biomech. 41:711-714. https://doi.org/10.1016/j.jbiomech.2007.09.029.

Bey, M. J., R. Zauel, S. K. Brock, and S. Tashman. 2006. Validation of a new model-based tracking technique for measuring three-dimensional, in vivo glenohumeral joint kinematics. J. Biomech. Eng. 128:604-609. https://doi.org/10.1115/1.2206199.

Bicalho, R. C., L. D. Warnick, and C. L. Guard. 2008. Strategies to analyze milk losses caused by diseases with potential incidence throughout the lactation: A lameness example. J. Dairy Sci. 91:2653-2661. https://doi.org/10.3168/jds.2007-0744.

Blackie, N., E. C. L. Bleach, J. R. Amory, and J. R. Scaife. 2013. Associations between locomotion score and kinematic measures in dairy cows with varying hoof lesion types. J. Dairy Sci. 96:35643572. https://doi.org/10.3168/jds.2012-5597.

Brainerd, E. L., D. B. Baier, S. M. Gatesy, T. L. Hedrick, K. A Metzger, S. L. Gilbert, and J. J. Crisco. 2010. x-ray reconstruction of moving morphology (XROMM): Precision, accuracy and applications in comparative biomechanics research. J. Exp. Zool. A Ecol. Genet. Physiol. 313:262-279. https://doi.org/10.1002/jez .589 .

Burow, E., P. T. Thomsen, T. Rousing, and J. T. Sørensen. 2014 Track way distance and cover as risk factors for lameness in Danish dairy cows. Prev. Vet. Med. 113:625-628. https://doi.org/10 .1016/j.prevetmed.2013.11.018.

Cook, N. B., and K. V. Nordlund. 2009. The influence of the environment on dairy cow behavior, claw health and herd lameness dynamics. Vet. J. 179:360-369. https://doi.org/10.1016/j.tvjl.2007 .09 .016 .

Crisco, J. J., and R. D. McGovern. 1998. Efficient calculation of mass moments of inertia for segmented homogeneous three-dimensional objects. J. Biomech. 31:97-101. https://doi.org/10.1016/S0021 -9290(97)00108-5.

Ehlert, A. 2006: Röntgenanatomische und querschnittsanatomische Untersuchungen unter Berücksichtigung magnetresonanztomographischer Befunde an der Hintergliedmaße des Rindes. Faculty of Veterinary Medicine, Leipzig.

El-Shafaey, El-S. A., T. Aoki, M. Ishii, and K. Yamada. 2013. Pilot study of bovine interdigital cassetteless computed radiography J. Vet. Med. Sci. 75:1503-1506. https://doi.org/10.1292/jvms.13 $-0240$

Gatesy, S. M., D. B. Baier, F. A. Jenkins, and K. P. Dial. 2010. Scientific rotoscoping: A morphology-based method of 3-D motion analysis and visualization. J. Exp. Zool. A Ecol. Genet. Physiol. 313:244-261. https://doi.org/10.1002/jez.588.

Geiger, S. M., E. Reich, P. Böttcher, S. Grund, and J. Hagen. 2017. Validation of biplane high-speed fluoroscopy combined with two different non-invasive tracking methodologies for measuring in vivo distal limb kinematics of the horse. Equine Vet. J. http://dx .doi.org/https://doi.org/10.1111/evj.12717.

Giphart, J. E., C. A. Zirker, C. A. Myers, W. W. Pennington, and R. F. LaPrade. 2012. Accuracy of a contour-based biplane fluoroscopy technique for tracking knee joint kinematics of different speeds. J. Biomech. 45:2935-2938. https://doi.org/10.1016/j.jbiomech .2012 .08 .045
Green, L. E., V. J. Hedges, Y. H. Schukken, R. W. Blowey, and A. J. Packington. 2002. The impact of clinical lameness on the milk yield of dairy cows. J. Dairy Sci. 85:2250-2256. https://doi.org/10 .3168/jds.S0022-0302(02)74304-X.

Henderson, S. E., R. Desai, S. Tashman, and A. J. Almarza. 2014 Functional analysis of the rabbit temporomandibular joint using dynamic biplane imaging. J. Biomech. 47:1360-1367. https://doi .org/10.1016/j.jbiomech.2014.01.051.

Knoerlein, B. J., D. B. Baier, S. M. Gatesy, J. D. Laurence-Chasen, and E. L. Brainerd. 2016. Validation of XMALab software for marker-based XROMM. J. Exp. Biol. https://doi.org/10.1242/jeb .145383 .

Main, D. C. J., K. A. Leach, Z. E. Barker, A. K. Sedgwick, C. M. Maggs, N. J. Bell, and H. R. Whay. 2012. Evaluating an intervention to reduce lameness in dairy cattle. J. Dairy Sci. 95:2946-2954. https://doi.org/10.3168/jds.2011-4678.

McDonald, C. P., C. C. Bachison, V. Chang, S. W. Bartol, and M. J. Bey. 2010. Three-dimensional dynamic in vivo motion of the cervical spine: assessment of measurement accuracy and preliminary findings. Spine J. 10:497-504. https://doi.org/10.1016/j.spinee 2010.02.024.

Meyer, S. W., M. A. Weishaupt, and K. A. Nuss. 2007. Gait pattern of heifers before and after claw trimming: A high-speed cinematographic study on a treadmill. J. Dairy Sci. 90:670-676. https://doi .org/10.3168/jds.S0022-0302(07)71549-7.

Miranda, D. L., J. B. Schwartz, A. C. Loomis, E. L. Brainerd, B. C. Fleming, and J. J. Crisco. 2011. Static and dynamic error of a biplanar videoradiography system using marker-based and markerless tracking techniques. J. Biomech. Eng. 133:121002. https://doi .org/10.1115/1.4005471.

Nuss, K., C. Sauter-Louis, and B. Sigmund. 2011. Measurements of forelimb claw dimensions in cows using a standardised sole thickness: A post-mortem study. Vet. J. 190:84-89. https://doi.org/10 $.1016 / j . t v j l .2010 .10 .002$.

Ocal, M. K., F. Sevil, and U. Parin. 2004. A quantitative study on the digital bones of cattle. Ann. Anat. 186:165-168. https://doi.org/ 10.1016/S0940-9602(04)80034-7.

Panagiotopoulou, O., J. W. Rankin, S. M. Gatesy, and J. R. Hutchinson. 2016. A preliminary case study of the effect of shoe-wearing on the biomechanics of a horse's foot. PeerJ 4:e2164 https://doi .org/10.7717/peerj.2164.

Schmid, T., M. A. Weishaupt, S. W. Meyer, N. Waldern, K. von Peinen, and K. Nuss. 2009. High-speed cinematographic evaluation of claw-ground contact pattern of lactating cows. Vet. J. 181:151157. https://doi.org/10.1016/j.tvjl.2008.02.019.

Tashman, S., and W. Anderst. 2003. In vivo measurement of dynamic joint motion using high speed biplane radiography and CT: Application to canine ACL deficiency. J. Biomech. Eng. 125:238-245. https://doi.org/10.1115/1.1559896.

Wachs, K., M. S. Fischer, and N. Schilling. 2016. Three-dimensional movements of the pelvis and the lumbar intervertebral joints in walking and trotting dogs. Vet. J. 210:46-55. https://doi.org/10 $.1016 /$ j.tvjl.2015.12.009.

Warnick, L. D., D. Janssen, C. L. Guard, and Y. T. Gröhn. 2001. The effect of lameness on milk production in dairy cows. J. Dairy Sci 84:1988-1997. https://doi.org/10.3168/jds.S0022-0302(01)74642 $-5$.

You, B. M., P. Siy, W. Anderst, and S. Tashman. 2001. In vivo measurement of 3D skeletal kinematics from sequences of biplane radiographs: Application to knee kinematics. IEEE Trans. Med. Imaging 20:514-525. https://doi.org/10.1109/42.929617. 